\title{
Clinical studies of the Tildox AVZ complex drug efficacy in respiratory and gastrointestinal pathologies of young pigs caused by opportunistic microflora
}

\author{
Sergey V. Engashev ${ }^{1}$, Aleksey V. Savinkov,2,*, Konstantin M. Sadov², Pavel V. Iliasov ${ }^{3}$ \\ ${ }^{1}$ Moscow State Academy of Veterinary Medicine and Biotechnology named after K.I. Skryabin, 109472, Moscow, Russian Federation \\ ${ }^{2}$ Samara State Agrarian University, 2 Uchebnaya str., Ust-Kinelsky settlement, Kinel, Samara Region, 446442, Russian Federation \\ ${ }^{3}$ Institute for Experimental Medicine and Biotechnologies, Samara State Medical University, Ministry of Health of the Russian \\ Federation, 20 Gagarina str., Samara, 443079, Russian Federation
}

\begin{abstract}
This publication provides a study of the efficacy of Tildox AVZ (a combined formulation of doxycycline and tylosin) in non-specific bacterial respiratory and gastrointestinal pathologies in piglets. The study population included 70 piglets with respiratory and gastrointestinal pathologies randomized into 6 groups. The effects of investigational drug used according 3-day or 5-day regimen were assessed using clinical examination, as well as bacteriological, blood count and blood chemistry lab tests. The investigational drug (Tildox AVZ) was safe for target animals and had no adverse effects, showed a similar species specificity and efficacy on the respiratory and gastrointestinal flora in piglets vs. the reference drug (Gentamicin) and allows achieving a beneficial clinical effect in respiratory and gastrointestinal diseases caused by opportunistic microflora. A significant clinical effect is achieved by Day 4 in gastrointestinal pathology and by Days 6-7 in respiratory pathology. Moreover, the control group with gastrointestinal pathology had no changes in the most of the parameters vs. baseline even on Day 7. Tildox AVZ had no adverse effects on blood count and blood chemistry parameters; on the contrary, for a number of parameters, a trend towards normalization was noted. The investigational drug was at least equivalent to the reference one (Gentamicin) and can be used for both respiratory and gastrointestinal disorders caused by the non-specific opportunistic bacterial microflora.
\end{abstract}

\section{Introduction}

Bacterial diseases of livestock, in particular pigs, are globally spread and cause great economic losses due to animal mortality and low weight gain ${ }^{1-6}$. In this regard, NVC Agrovetzashchita LLC has developed Tildox AVZ, a drug for use in bacterial respiratory and gastrointestinal diseases of pigs. To perform an objective assessment of the efficacy and safety of the drug, its efficacy against respiratory and gastrointestinal pathogens must be proven by clinical and bacterial diagnostics methods, blood tests, and general status of animals.

Tildox AVZ is a light yellow powder; it belongs to combined antibacterial drugs. It contains doxycycline and tylosin as active ingredients. Doxycycline is a semisynthetic tetracycline antibiotic active against Grampositive and Gram-negative microorganisms, including: Bordetella spp., Campylobacter spp., Escherichia coli, Haemophilus spp., Pasteurella spp., Salmonella spp., Staphylococcus spp., Streptococcus spp., Clostridium perfringens, Chlamydia spp., Rickettsia spp., as well as Mycoplasma spp. ${ }^{7,8}$. The bacteriostatic effect of doxycycline is based on inhibition of the enzymes catalyzing binding of aminoacetyl tRNA to ribosomal acceptors, which disrupts protein synthesis and leads to the death of a microbial cell.

Tylosin is a macrolide antibiotic active against most Gram-positive and some Gram-negative bacteria, including Staphylococcus spp., Streptococcus spp., Bacillus anthracis, Corynebacterium spp., Clostridium spp., Pasteurella spp., Erysipelothobacterium spp., Fchaerotespp. spp., Leptospira spp., Treponema hyodysenteriae, Actinomyces pyogenes, Haemophilus pertussis, Moraxella bovis, Neisseria spp. ${ }^{9,10}$. The bacteriostatic effect of tylosin is based on blocking the protein synthesis in a bacterial cell by binding $50 \mathrm{~S}$ ribosomal subunit.

When administered orally, the antibiotics are well absorbed in the gastrointestinal tract and enter into most organs and tissues of the animal, reaching maximum serum concentrations in 1.5-2 hours ${ }^{11}$. Doxycycline and tylosin are metabolized to an insignificant extent, being eliminated from the body mainly with bile and urine ${ }^{12,13}$.

Tildox AVZ belongs to moderately hazardous substances (hazard class 3 according to GOST 12.1.007) as assessed by its effects in animal body. It is well tolerated by animals in recommended doses and possess no hepatotoxicity, embryotoxicity and teratogenic properties.

\footnotetext{
Corresponding author: sever291@ mail.ru
} 
The aim of this study was to investigate the Tildox AVZ efficacy for veterinary use in pig diseases caused by microorganisms sensitive to doxycycline and tylosin. To investigate the safety and identify possible adverse effects of Tildox AVZ when administered to pigs.

Objectives of the present study included:

treatment of young pigs with clinical signs of respiratory and gastrointestinal diseases in 3-day and 5day regimen arms;

follow-up of the animals until Day 20 while assessing the clinical status of the animals and performing bacteriology, hematology and blood chemistry tests.

\section{Materials and Methods}

Clinical studies were conducted from April 23, 2019 to May 15, 2019 on 70 piglets aged 35-45 days belonging to Myasoagroprom LLC, Krasnoyarsk district, Samara region. The laboratory tests were performed in the Samara Regional Veterinary Laboratory State Budgetary Institution of the Samara Region and Samara State Medical University of Ministry of Health of the Russian Federation, IVD Microbiological Department of SamSMU clinical hospital, SamBio LLC.

Total of 70 piglets were randomized into six groups. The effect of the drug was evaluated in animals with respiratory and gastrointestinal pathologies, and for each of the two pathology types, 3 groups were formed: control; treatment using 3 day regimen; and treatment using 5 day regimen.

Tildox AVZ was administered orally as a solution by the group method with water $(1 \mathrm{~g}$ of the drug per $10 \mathrm{~kg}$ of animal weight) for 3 and 5 days in the respective groups.

Control animals did not receive Tildox AVZ but were administered with Gentamicin powder (KRKA) approved in the Russian Federation in accordance with the established procedure.

All animals during the experiment were in the same housing and feeding conditions.

Tildox AVZ efficacy was assessed using:

- monitoring of clinical signs of the disease, disappearance or decrease in the clinical signs in treatment groups vs. control group. Clinical examination included measurements of body temperature, food consumption, physical activity and the appearance of the animals. Meanwhile, food consumption was assessed using a 4-point scale, where 0 corresponded to food refusal, 1 to poor, 2 to moderate and 3 to normal consumption. The physical activity and appearance were assessed as binary variables, where 0 corresponded to reduced activity or "unsatisfactory" appearance, and 1 to normal activity or "satisfactory" appearance, respectively;

- bacteriological study of swabs from the rectum and nasal passages. Evaluation of the reduction in or elimination of the most active forms of opportunistic bacterial flora in the treatment and control groups;
- blood count. Evaluation of the decrease in the reactive manifestations of the inflammatory process in the treatment groups vs. controls;

- blood chemistry tests. Recording changes in blood chemistry during the recovery of piglets in the treatment and control groups;

- statistical processing of the data obtained. For each time point, parameter and group, we calculated the mean and standard deviation for quantitative variables, the median and interquartile range for rank variables, and the proportion and error of the proportion for binary variables, and assessed the dynamics of the corresponding parameters. Spearman's rank correlation coefficient was used to compare the dynamics of various parameters. Data were compared between groups using Student's t-test for quantitative variables, provided that the data met the normal distribution, Wilcoxon-MannWhitney test for rank variables and $\chi 2$ test for binary variables. The results were considered significant at $\mathrm{p}<$ 0.05 .

The lack of signs such as diarrhea, depression, refusal of feed for piglets with gastrointestinal pathology, and cough, nasal discharge, characteristic forced postures, refusal to feed, depression for piglets with respiratory pathology was regarded as clinical wellbeing.

The lack of an adverse drug reaction in the treatment groups, including skin irritation, anxiety, itching, swelling, hyperemia, salivation, allergic reactions suggested that the drug has no adverse effects on pigs.

\section{Results}

Tildox AVZ efficacy in the respiratory pathology of piglets.

At the baseline, the following representatives of opportunistic flora were found in nasal washings in piglets with respiratory pathology: S. saprophyticus, Klebsiella sp., Streptococcus sp., S. epidermidis, and Candida sp. Klebsiella sp. were the most common opportunistic bacteria. (83.3\%), and S. epidermidis had the minimum prevalence $(19.4 \%)$.

During treatment, Tildox AVZ showed activity against Staphylococcus sp., Candida sp., Streptococcus sp., Klebsiella sp. E. coli appeared during the experiment, and the effect of Tildox AVZ and Gentamicin on this microorganism was not fully understood. At the same time, the changes in flora was similar in Groups 1 and 1.1 (correlation coefficient 0.7, p $>0.95$ ), while significantly differing from the control group. In both treatment groups, the correlation with the baseline level still remained (correlation coefficients 0.87 and $0.81, p>0.95$ ). In terms of the frequency and differences between groups, we cannot claim that the drug has more marked effect on any opportunistic microorganism; however, an increased effect of investigational treatment on Staphylococcus sp. and Citrobacter sp. vs. control can be noted. In general, the findings reflected a more uniform effect of Tildox AVZ on all studied microorganisms vs. the standard treatment regimen. 
On Days 10 and 11, populations of all the microorganisms except for Candida sp., showed a trend to recovery in groups 1 and 1.1. Neither the investigational nor the reference treatment regimen caused elimination of any of the microorganisms commonly found in sick animals at the baseline. Considering that by this time point the piglets were clinically healthy, the existing microflora becomes the resident one and does not have a marked pathogenic effect.

In general, the assessment of the clinical effect of the investigational drug showed that it allowed normalizing the animals' status by 4 parameters (body temperature, food consumption, physical activity and appearance) (Fig. 1).

At the same time, there were no significant differences between the treatment groups, and the differences between the treatment and control groups were only registered for "feed consumption" parameter
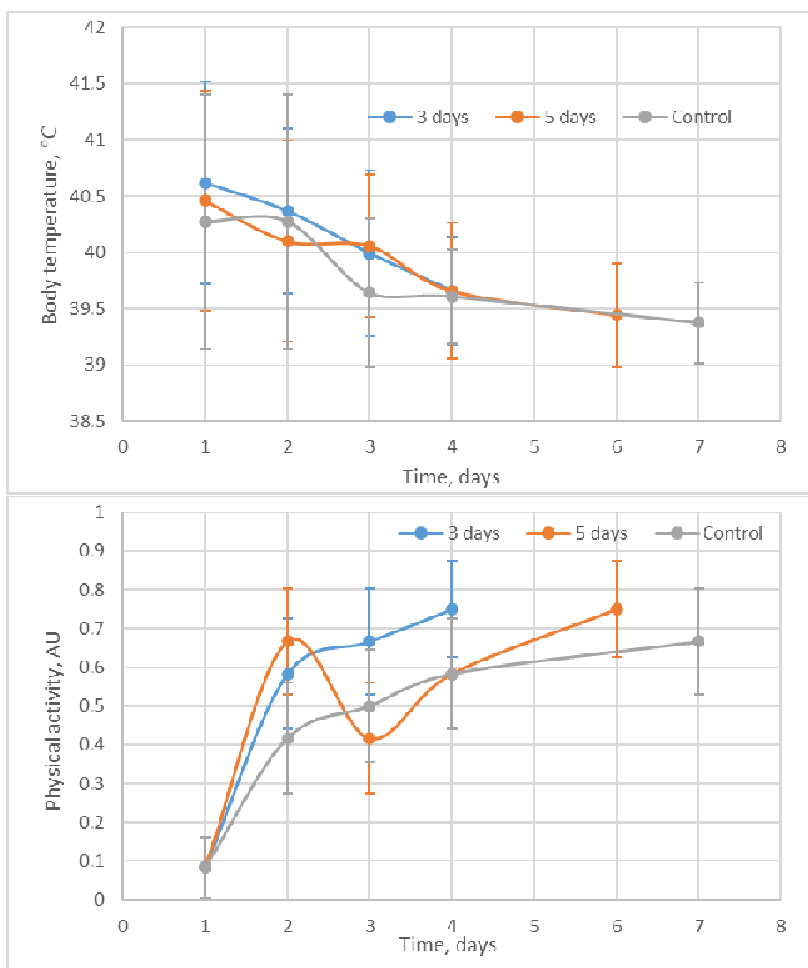

on Day 2. Comparison of the dynamics of the assessed parameters revealed their fuzzy correlation to each other in the treatment groups, although all the parameters showed similar dynamics in the control group.

Similarly, comparison of the dynamics of each indicator between groups showed that such a correlation takes place only for body temperature and the "appearance" parameter in all groups, as well as for food consumption between Groups 1 and 2 and for physical activity between Group 1 and the control group; but in all other cases, $\mathrm{p}$ value exceeded 0.05 .

It should be noted that despite the obvious trend of the parameters to normalization, significant differences by Day 3 vs. baseline were only observed for body temperature and physical activity in Group 1 (3-day treatment regimen), for body temperature in group 1.1 (5-day treatment regimen) and for food consumption in the control group. The remained parameters in other groups only showed such changes by Days 6-7.
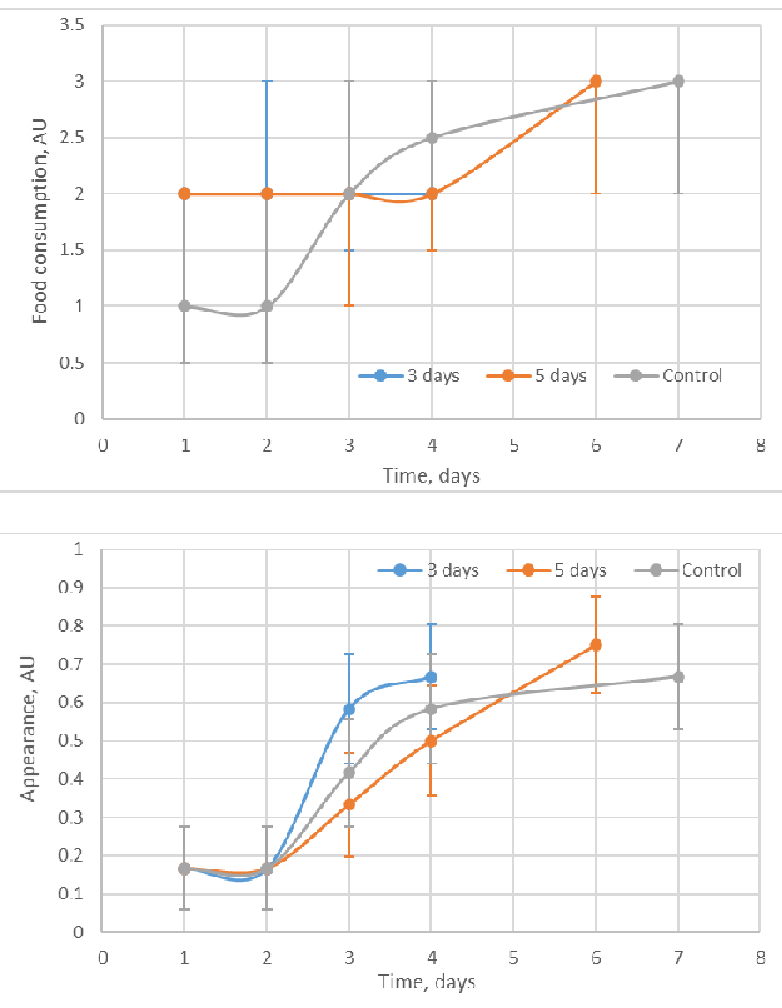

Fig. 1. Dynamics of clinical status of animals with respiratory pathology.

On Days 10 and 21, the piglets in treatment Groups 1 and 1.1 and in the control group had no signs of the disease at all, had good appetite and physical activity, and no deviations in appearance (data not shown).

Evaluation of the effect of the investigational drug on hematology showed that by Day 10, there were significant changes in the WBC, hemoglobin level, percentage of segmented neutrophils and lymphocytes vs. baseline in both treatment and control groups, which remained up to 20 days of follow-up. With regard to the blood chemistry, changes were observed in the levels of creatinine in the treatment and control groups, alkaline phosphatase in the treatment groups, and urea in the control group. By the end of follow-up, changes in the total protein level also appeared in the treatment and control groups.

Tildox AVZ efficacy in the gastrointestinal pathology of piglets

At the baseline, E. coli was inoculated in rectal swabs of piglets having gastrointestinal pathology with diarrheal syndrome (E. coli was not typed for pathogenicity). In about half of the cases, yeast microflora (Candida sp.) was detected. In addition, Streptococcus sp. and, in a minority of cases, Klebsiella sp. were found (5.6\%). Subsequently, Staphylococcus sp. and Proteus sp. were appended in a number of animals. 
During the treatment, there was a general moderate correlation between changes in flora in the treatment and control groups (the correlation coefficient is $0.71, \mathrm{p}=$ 0.95). Similarly to the respiratory pathology, after the discontinuation of the drug both the treatment and control groups showed a trend towards recovery of the populations of most microorganisms, except for Candida sp. and Staphylococcus sp. At the same time, the prevalence of E. coli and Streptococcus sp. by Day 11 days was comparable to the baseline levels (E. coli) or exceeded them (Streptococcus sp. in the treatment groups).

These bacteria were probably resident and quite resistant to antibacterial drugs. Meanwhile, both investigational and control regimens gave a comparable changes in microflora and eliminated Candida sp. and Staphylococcus sp. from the gastrointestinal tract of animal subjects, which, apparently, improved their clinical status. The remaining populations of other microorganisms did not appear to be significant pathogens and at least did not cause the diarrheal syndrome observed in the subjects at the baseline.

Evaluation of the clinical effect of Tildox AVZ in gastrointestinal pathology, similarly to the respiratory diseases, showed the lack of differences between the
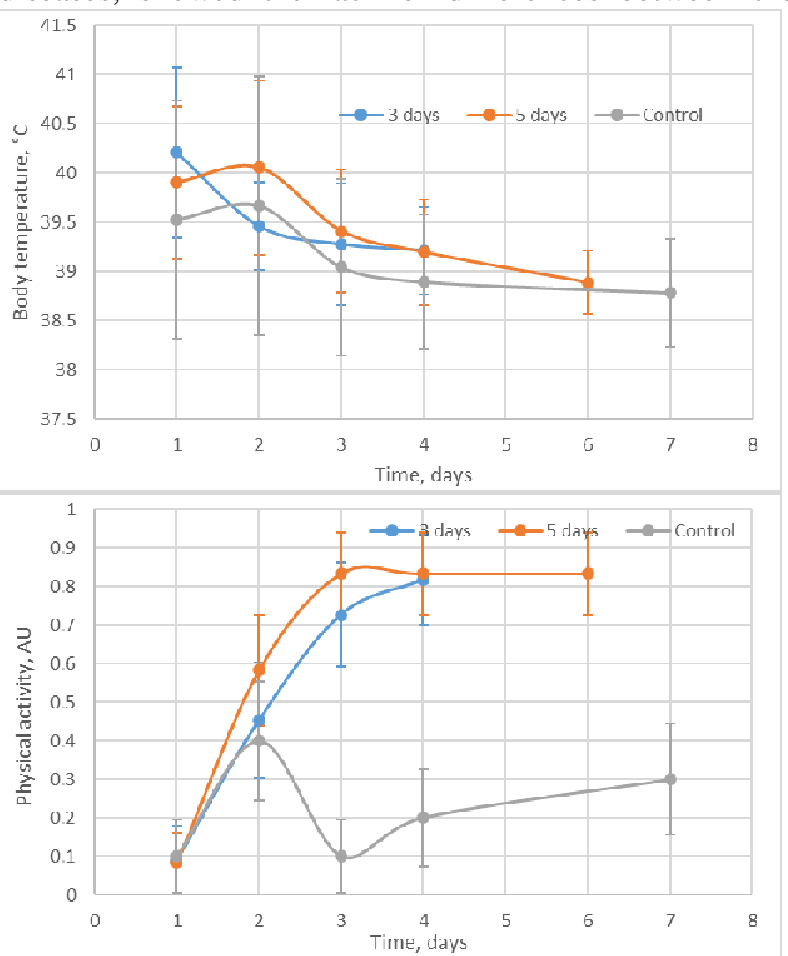

treatment groups, and the most significant systematic differences between the treatment and control groups for the "physical activity" parameter since Day 3.

The correlation of the dynamics between the assessed parameters in all groups was also fuzzy, and the dynamics of each parameter between groups correlated only for the "appearance" parameter in all groups, body temperature between group 2.2 and control and motor activity between group 2 and the control; in all other cases, $\mathrm{p}$ value exceeded 0.05 .

At the same time, in contrast to the respiratory pathologies, almost all parameters in both treatment groups developed significant differences vs. baseline as early as by Day 4; as for the control group, all the parameters, except for food consumption, did not differ significantly from the baseline even by Day 7 (Fig. 2). The overall course of treatment of respiratory and gastrointestinal diseases was characterized by a fairly close similarity which was confirmed by a significant correlation between body temperature and the "appearance" parameter in all groups, "physical activity" parameter between 5-day treatment regimen and control groups, as well as "feed consumption" parameter between 3-day treatment regimen groups.

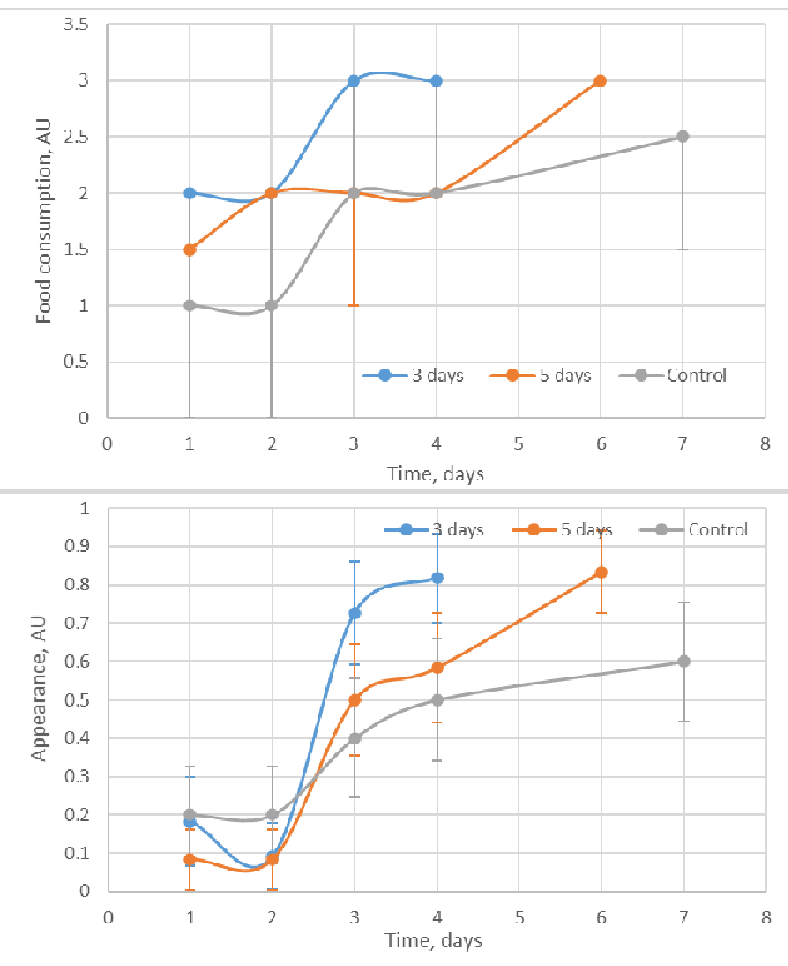

Fig. 2. Dynamics of clinical status of animals with gastrointestinal pathology.

On Days 10 and 21, similarly to respiratory diseases, the piglets in treatment Groups 2 and 2.2 and in the control group had no signs of the disease at all, had good appetite and physical activity, and no deviations in appearance (data not shown).

Hematology tests only showed significant changes vs. baseline for the eosinophils in the treatment and control groups, while other WBC differential parameters did not statistically differ from the baseline by the end of the study. In addition, there was almost 1.6-fold increase in the platelets in the treatment groups vs. baseline. The baseline WBC, in contrast to the respiratory pathology, were much closer to normal values, and their changes during the study were insignificant. Similarly, there were no significant changes in hemoglobin levels, although baseline values were even lower than in respiratory diseases.

As for blood chemistry, the most significant changes were noted for the serum glucose level, which significantly changed in the treatment groups as early as 
by Day 3. Later, by Day 10, such changes were noted for albumin, creatinine in both treatment and control groups, as well as for alkaline phosphatase in Group 2 and ALT in Group 2.2. The baseline urea and total protein levels in gastrointestinal pathologies were closer to normal than in respiratory diseases, and their changes during the study were insignificant.

\section{Conclusion}

The data obtained suggest that the investigational drug (Tildox AVZ) is safe for target animals and has no adverse effects, shows a similar species specificity and efficacy on the respiratory and gastrointestinal flora in piglets vs. the reference drug (Gentamicin) and allows achieving a similar beneficial clinical effect in respiratory and gastrointestinal diseases caused by opportunistic microflora. A significant clinical effect is achieved by Day 4 in gastrointestinal pathology and by Days 6-7 in respiratory pathology. Moreover, the control group with gastrointestinal pathology had no changes in the most of the parameters vs. baseline even on Day 7. Neither Tildox AVZ nor Gentamicin had adverse effects on hematology and blood chemistry; on the contrary, for a number of parameters, a trend towards normalization was noted. Thus, the investigational drug is not inferior to the reference one and can be used to treat respiratory and gastrointestinal pathologies caused by nonspecific opportunistic bacterial microflora.

\section{References}

1. D.E. Te Beest, T.J. Hagenaars, J.A. Stegeman, M.P. Koopmans, M. van Boven, Veterinary research, 42, 1 (2011)
2. E. van Duijkeren, B. Catry, C. Greko, M.A. Moreno, M.C. Pomba, S. Pyorala, M. Ruzauskas, P. Sanders, E.J. Threlfall, J. Torren-Edo, K. Torneke, The Journal of antimicrobial chemotherapy, 66, 12 (2011)

3. K. Wilkinson, W.P. Grant, L.E. Green, S. Hunter, M.J. Jeger, P. Lowe, G.F. Medley, P. Mills, J. Phillipson, G.M. Poppy, J. Waage, Philosophical transactions of the Royal Society of London Series

B, Biological sciences, 366, 1573 (2011)

4. E. Crisci, T. Mussa, L. Fraile, M. Montoya, Molecular immunology, 55, 3-4 (2013)

5. Q. Zhang, R. Hu, X. Tang, C. Wu, Q. He, Z. Zhao, H. Chen, B. Wu, Archives of virology, 158, 8 (2013)

6. E. Reynaga, C. Torres, M. Garcia-Nunez, M. Navarro, A. Vilamala, E. Puigoriol, G.E. Lucchetti, M. Sabria, Clin. Microbiol. Infect., 23, 9 (2017)

7. G. Pulverer, Med. Klin., 64, 22 (1969)

8. J.P. Butzler, Chemotherapy, 21 Suppl 1 (1975)

9. S. Ota, A. Hamagami, Y. Terawaki, R. Nakaya, Jpn. J. Antibiot., 23, 2 (1970)

10. R. Okamoto, M. Tsuchiya, H. Nomura, H. Iguchi, K. Kiyoshima, S. Hori, T. Inui, T. Sawa, T. Takeuchi, H. Umezawa, J. Antibiot. (Tokyo), 33, 11 (1980)

11. T. Avci, M. Elmas, The Scientific World Journal (2014)

12. L. Brunton, B.A. Chabner, B. Knollman, Goodman and Gilman's The Pharmacological Basis of Therapeutics, Twelfth Edition (McGraw-Hill Education, New York, 2011)

13. D.E. Golan, E.J. Armstrong, A.W. Armstrong, H. Wolters Kluwer, Principles of pharmacology: the pathophysiologic basis of drug therapy (Wolters Kluwer, Philadelphia [ect.], 2017) 